\title{
ESTUDIO DE NECESIDADES Y PERFIL SOCIO DEMOGRÁFICO DE LOS PADRES DE ESTUDIANTES DE NUEVO INGRESO DEL AÑO ACADÉMICO 2009-2010
}

\author{
Georgina Bueno Delgado' \\ Maritza I. Pérez Rodríguez ${ }^{2}$ \\ Carol Y. Irizarry Robles ${ }^{3}$
}

Se considera necesario, que la Universidad haga un esfuerzo por mejorar los servicios a los estudiantes y que se cultive la autoestima, la confianza es sí mismo, la iniciativa, el liderazgo, la independencia de criterio y la prudencia en la toma de decisiones, capacitándolos para hacer juicios éticos, anticipar problemas, percibir oportunidades y proponer cambios constructivos, proporcionando así el desarrollo integral y equilibrado de la persona

(Ostolaza, 2001, p.170).

\section{Resumen}

La familia es el primer laboratorio en el que el estudiante empieza a relacionarse positiva o negativamente con otros seres humanos. Es la agencia socializadora más importante en su desarrollo y los patrones de crianza y las características de su familia de origen pueden ejercer una gran influencia en su conducta futura. Por lo tanto, las relaciones familiares y la preparación académica de sus padres, entre otros, impactan su éxito o fracaso en los estudios. Es meritorio que los profesionales de ayuda (trabajadores sociales, consejeros profesionales y psicólogos), que ofrecen apoyo y ayuda al estudiantado, conozcan su trasfondo familiar y sus características socio demográficas para estar en mejor posición de ayudarlos a atender sus necesidades y a lograr sus metas académicas y personales. Este artículo presenta los resultados

\footnotetext{
'Trabajadora social en el Departamento de Consejería para el Desarrollo Estudiantil del Decanato de Estudiantes de la Universidad de Puerto Rico en Río Piedras.

${ }^{2}$ Consejera Departamento de Consejería para el Desarrollo Estudiantil del Decanato de Estudiantes de la Universidad de Puerto Rico en Río Piedras

${ }_{3}^{3}$ Estudiante doctoral de Psicología en la Universidad de Puerto Rico en Río Piedras.
} 
del estudio Necesidades y Perfil Socio Demográfico de los Padres de Estudiantes de Nuevo Ingreso del Año Académico 2009-2010 realizado en el Recinto de Río Piedras de la Universidad de Puerto Rico. Se realiza un análisis descriptivo y se establecen correlaciones entre el perfil socio demográfico de los padres y los hijos que inician estudios universitarios; también se documentan las necesidades más apremiantes que presentan estos padres en torno a sus hijos. [Descriptores: Estudiantes universitarios de nuevo ingreso, padres de estudiantes universitarios, necesidades de los padres de hijos universitarios, datos socio demográficos de padres de estudiantes universitarios, datos socio demográficos de estudiantes universitarios].

\section{Abstract}

The family is the first scenario in which students begin to relate positively or negatively with other human beings. It is the most important socializing agency contributing to his and her development. Upbringing patterns and family of origin characteristics exert a great influence in the student's future behavior. Family relationships and the parents' academic standing, among other factors, have a great deal to do with success or failure in their studies. It is important that helping professionals (social workers, professional counselors, and psychologists) get to know the students' family history and background, as well as the family's socio-demographic characteristics in order to help them better attend to their needs and achieve their personal and academic goals. This article presents the findings of the research Needs assessment and socio-demographic profile of parents of new students in the academic year 2009-2010, performed at the Río Piedras Campus of the University of Puerto Rico. A descriptive analysis was done as well as the correlations between parents' socio-demographic profile and the students starting college. As an added contribution, the article documents the most immediate needs these parents face regarding their children.[Keywords: first year college students, college students' parents, needs of college students' parents, socio-demographic data of college students' parents, college students' socio-demographic data].

\section{Introducción}

En 1943 se funda el Decanato de Estudiantes de la Universidad de Puerto Rico del Recinto de Río Piedras como junta de servicio al estudiante, encaminado al logro de la misión general de la Universidad. Este decanato, expone Rivera (2003), tiene la responsabilidad de proveer servicios esenciales de calidad que propicien el bienestar del estudiante y promuevan la formación integral de ciudadanos que contribuyan al desarrollo 
de la sociedad. Atiende necesidades académicas, económicas, emocionales, físicas, sociales, y culturales. Al ofrecer estas ayudas se propone que el estudiante pueda alcanzar sus metas académicas en un ambiente que propicie su formación integral.

Para cumplir con esta responsabilidad, el Decanato de Estudiantes cuenta con 23 diferentes programas de servicio directo al estudiantado. Uno de ellos es el Departamento de Consejería para el Desarrollo Estudiantil, (DCODE), el cual ofrece servicios de orientación y consejería en el área educativa, vocacional, personal, social y familiar, de manera que el estudiante pueda alcanzar su óptimo desarrollo como educando y como persona (Rivera, 2003). EI DCODE, ofrece ayuda a la población estudiantil a nivel individual, grupal y en actividades masivas. Cuenta con docentes de la consejería en las áreas de Trabajo Social, Consejería y Psicología.

De otra parte, los estudiantes aceptados a la Universidad son aquellos que obtuvieron índices altos de aprovechamiento académico en la escuela superior y en el examen administrado por la Oficina del "College Board". Esto cumple con uno de los objetivos de la Universidad de promover el reclutamiento activo de estudiantes talentosos, particularmente procedentes de familias con escasos recursos económicos (Universidad de Puerto Rico, 1989-90). Este mismo objetivo también propone el ofrecer apoyo para la retención de estos estudiantes.

Sin embargo, a pesar de que los estudiantes admitidos han demostrado la capacidad intelectual para salir bien en los estudios y de que existen unos programas de apoyo académico para ayudarles en sus dificultades, una gran cantidad de estudiantes son puestos en probatoria, otros no obtienen el progreso académico deseado, otros no cumplen con los requisitos mínimos de retención y otros abandonan los estudios. Sostiene De Jesús (1988), que la suspensión de estudiantes debido a no alcanzar el progreso académico satisfactorio, podría plantear el incumplimiento por parte de esta institución pública universitaria de la encomienda que la sociedad puertorriqueña le ha asignado de adiestrar y preparar, de manera óptima, los recursos profesionales en las distintas disciplinas que aportan a su desarrollo y progreso.

De otra parte, según Navas (1998), dependerá de que el estudiante mantenga un nivel apropiado de ejecución según las evaluaciones del profesor quien refuerza la labor satisfactoria con una buena nota y la deficiente con una baja calificación. Al hablar de ejecución, Navas se refiere al comportamiento humano enfatizando que éste no se da en el vacío y que tanto las conductas aceptables como las no deseables ocurren en 
un contexto que se puede identificar y por ende se puede tener un panorama más amplio de cuándo, dónde y por qué ocurre la conducta. Señala que se pueden establecer los niveles de predicción de la futura ocurrencia de conocimiento de los eventos que han sucedido previos a dicho comportamiento y los eventos que han ocurrido después. Por consiguiente, el estudiante al ingresar a la universidad, aunque reúna los requisitos de admisión, esté motivado y posea los ingredientes básicos para el aprendizaje, no deja de desvincularse de su entorno familiar, social y cultural en el que ha crecido y se ha desarrollado. Ese equipaje lo acompañará a través de su vida universitaria. Ahi conserva las herramientas aceptables como las no deseables que ha aprendido en su hogar para enfrentar las situaciones del diario vivir. Si sus relaciones de familia y experiencias con ésta han sido positivas y saludables, es muy probable que le sirvan de base para desarrollar nuevas amistades, establecer buenas relaciones con sus profesores y su entorno; además contará con buenas redes de apoyo de sus familiares y allegados. Por el contrario, si carece de estas buenas relaciones y ha crecido en un ambiente de conflictos familiares, estará carente de fortalezas para desarrollar ese sentido de utilidad, pertenencia y poderío necesarios para un ajuste saludable a la vida universitaria y un buen rendimiento académico.

Al ser la familia la primera institución que provee lazos afectivos al estudiante, cuando éstos se afectan, se le trastocan las redes de apoyo de las principales figuras significativas en su vida. Como consecuencia, un estudiante afectado por dificultades familiares se le puede afectar también su concentración, memoria, motivación e interés en los estudios, relaciones con los pares, salud física y emocional, entre otros, lo que en la mayoría de los casos repercute en un pobre rendimiento académico. He ahí la urgencia de conocer las necesidades que confrontan los padres en relación a sus hijos universitarios de manera que se les provean las herramientas con las cuales puedan apoyarlos más efectivamente en el logro de sus metas académicas y en su desarrollo integral.

\section{Revisión de Literatura}

La buena comunicación con los padres y el apoyo que reciban de éstos son dos factores trascendentales en el logro de las metas académicas de los estudiantes. Kenny y Rice (1995) plantean que existe una correlación positiva entre el ajuste exitoso de los estudiantes de primer año de universidad y el vínculo sostenido con sus padres. A su vez, Puente (1999) sostiene que "en ocasiones los estudiantes fracasan no porque carezcan de estrategias cognitivas, sino porque carecen de 
estrategias afectivas de apoyo para desarrollar y mantener un estado psicológico interno y un ambiente de aprendizaje apropiado" (pág. 283).

En un estudio realizado por Hummel y Steele (1996) se encontró que los estudiantes que reciben apoyo, motivación y ayuda de sus padres en el logro de sus metas académicas, no sólo perseveran sino que experimentan un intenso deseo de sobresalir en los estudios y de superar su nivel de vida. De otra parte, Ruther (1999) expone que los jóvenes que mantienen buenas relaciones familiares están propensos a mantenerse en la universidad porque reciben de éstos apoyo emocional y ayuda en sus dificultades personales y en las decisiones acerca de sus carreras. Esto hace la diferencia entre aquellos que no tienen familia, o aquellos que si la tienen, no reciben apoyo de ella.

Algunos estudiantes al iniciar sus estudios universitarios y encontrarse lejos de sus familias, pueden experimentar inseguridad y ansiedad, en parte porque no han recibido de sus padres el impulso necesario para separarse de ellos. Además, durante el proceso de esa transición se ven obligados a hacer muchos ajustes en un período de tiempo corto caracterizado por las expectativas académicas de la universidad y los propios padres.

Argumentan Whitaker y Slimak (1993) que en la etapa inicial de estudios universitarios hay tres procesos relacionados entre sí que interfieren en su desarrollo y éxito académico, los cuales se caracterizan por un individualismo relativo, un proceso de lealtad y el ciclo de vida familiar. En estos procesos hay muchas variables que intervienen como la intensidad de la dependencia entre el estudiante y su familia, la flexibilidad de sus padres, la separación geográfica y las consideraciones económicas. Según el individualismo relativo que proponen, la familia considera que la separación va y viene en forma relativa manteniendo unos lazos de unión y afecto en los que el estudiante experimenta presión para salir del seno familiar, alcanzar sus sueños y llenar unas necesidades. Al mismo tiempo siente la urgencia de estar cerca de la familia para cumplir con sus demandas inmediatas, como lo es proveerles apoyo emocional para mitigar esa separación.

En el ciclo de la vida familiar, argumentan estos autores, los cambios son rápidos; si los padres son flexibles y comprensivos en la necesidad de autonomía, identidad y separación de sus hijos, es menos probable que haya problemas. Si por el contrario, es una familia rígida y no acepta o comprende los diferentes cambios en el joven, se forma una reacción disfuncional donde éste no logra apoyo ni entendimiento en esta transición. En algunos casos se espera que el hijo siga la tradición de estudiar las carreras de sus padres, como sucede en las familias enteras 
de médicos o de abogados. Cuando ocurre este fenómeno pueden surgir conflictos que resultan en bajo rendimiento del estudiante, frustración y desinterés, ya que deben estudiar una carrera que a veces no está a tono con sus destrezas, intereses, habilidades y características de personalidad. Palladino y otros (2001) realizaron un estudio sobre cómo las relaciones de familia influían en la toma de decisiones y selección de carreras, y encontraron que las experiencias de la niñez, el modelaje, el apoyo emocional, la estima, la información, la ayuda tangible, y la localización geográfica de los padres influían positivamente en la selección de carrera en los estudiantes.

Los estudiantes que no se integran social y académicamente a la universidad, argumenta Tinto (1999), son aquellos que no logran sobrepasar exitosamente la etapa de separación de su familia y la de transición e integración al ambiente universitario. De ahí que su transición y ajuste exitoso depende en gran medida del apoyo que reciban de sus padres. Sostiene este autor que el abandonar los estudios en su primer año de universidad no necesariamente tiene que ver con su incapacidad de integrarse a las comunidades sociales y académicas, sino más bien con su inhabilidad para lidiar con el estrés causado por la separación de su familia y de su comunidad. Al igual que Agliata (2005), Tinto sugiere que se deben realizar actividades para los estudiantes nuevos y sus padres tales como ofrecer talleres y distribuir opúsculos con el fin de educarlos sobre el proceso de separación y transición de sus hijos a la universidad, así como las normas, valores y sistemas de creencias que se espera que los estudiantes puedan adoptar. Enfatiza Tinto la importancia de propiciar la participación de los padres y el instarlos a apoyar y estar al lado de sus hijos en el proceso de ajuste institucional ya que eso les puede ayudar a triunfar.

Un estudio realizado por Carter (2006) con estudiantes de nuevo ingreso sustenta los postulados de Tinto (1999), ya que reflejó que la participación de los padres está relacionada al ajuste social y al logro académico de sus hijos, y que los estudiantes que confían en sus padres y están emocionalmente cercanos a ellos, tienen un mejor ajuste a la universidad. Además, encontró que el conflicto de los padres con sus hijos es una variable importante en el desarrollo del estudiante universitario. De igual forma, un estudio realizado por Hermann (2007) con estudiantes de primer año encontró que aquellos que tenían relaciones estrechas con sus padres obtuvieron un ajuste más exitoso a la universidad.

Estudios realizados sobre las actitudes de los estudiantes universitarios, sus logros académicos y su relación familiar reflejan que uno de los aspectos que más les preocupa es la presión 
que reciben de sus padres al no poder cumplir sus expectativas académicas, no obtener las calificaciones esperadas y no aceptar su comportamiento social (Whitaker \& Slimak, 1993; Whitaker, 1996). De igual forma sustenta Inghram (1998) que muchos jóvenes vienen a la universidad con reacciones tensas hacia su familia por aspectos relacionados con divorcios o matrimonios de sus padres, valores, sexo, dinero, selección de carreras y expectativas.

Estudios realizados con estudiantes universitarios buscaron examinar las discrepancias entre el desempeño académico y las percepciones de las expectativas de sus padres; éstos encontraron que los estudiantes reportaron niveles más altos de coraje, depresión, ansiedad, baja autoestima y dificultades de ajuste a la universidad cuando hubo mayores discrepancias entre expectativas y desempeño, según percibidas por los estudiantes (Agliata, 2005; Agliata \& Renk, 2008). Esto alerta sobre la necesidad de trabajar directamente con los estudiantes para crear metas realistas y formular planes para alcanzarlas; además, enfatiza la importancia de enseñar destrezas de comunicación asertiva a los estudiantes universitarios y a sus padres para disminuir las discrepancias entre las expectativas y las percepciones incorrectas.

De otra parte, la situación económica de los padres repercute en la vida del estudiante. Astin (1997) sostiene que este factor guarda relación en los universitarios con el desarrollo académico, el pensamiento crítico, las destrezas en la solución de problemas, las relaciones interpersonales y la terminación de una carrera. En sus investigaciones, Astin ha encontrado que aquellos estudiantes procedentes de familias de niveles socioeconómicos altos tienen más expectativas sobre sus logros académicos sin importar su habilidad, preparación académica u otras características. De igual forma argumenta Mohammdi (1996) que el factor económico de la familia prevalece siempre en estudiantes que aspiran a carreras. Un estudio Iongitudinal realizado por Horn y Kojaku (2001) también encontró que el factor económico de la familia estaba relacionado con la perseverancia en los estudios, ya que los estudiantes que provenían de familias de bajos ingresos tenían menos probabilidades de completar su currículo académico. También estudios realizados por García y San Segundo (2002) encontraron que las calificaciones de ingreso guardan relación con la situación socioeconómica del estudiante. Por otro lado, según Steinmayr, Dinger y Spinath (2010), parece haber una asociación entre la educación de los padres y el logro académico de los hijos; se ha observado que, a medida que aumenta la educación de los padres, aumenta también el porcentaje de estudiantes que completa su currículo 
(Horn \& Kojaku, 2001) y el bienestar psicológico de los estudiantes (Campbell, 2004), por lo que es esta variable debe ser explorada.

El Centro Nacional de Educación y Estadísticas de los Estados Unidos (Choy, 2002) realizó tres estudios longitudinales con el propósito de explorar la calidad de los estudiantes que ingresan a las universidades, la perseverancia en los estudios y su trayectoria una vez finalizaban sus carreras. Entre sus hallazgos, se encontró que aquellos estudiantes cuyos padres fueron a la universidad pueden manejar más efectivamente los retos de la vida universitaria resultando en un mejor ajuste, una mayor retención y tasas más altas de graduación. Entre otros hallazgos "el estudio concluyó que los estudiantes cuyos padres no poseen estudios universitarios están menos propensos a concluir una carrera universitaria" (Choy, 2002, p.29).

Se encontró que los que terminaban la escuela superior en un nivel básico o intermedio y cuyos padres no poseían estudios universitarios, tendían a obtener bajas calificaciones y estaban en desventaja si acudían a la universidad. Estos datos también fueron corroborados en escuelas superiores de alto prestigio. Una cuarta parte de estos estudiantes trabajaban a tiempo completo y tres cuartas partes trabajaban a medio tiempo o recibían algún tipo de ayuda económica. El estudio indicó que el riesgo mayor de abandonar los estudios estaba entre los estudiantes que trabajaban o que sus padres no poseían estudios universitarios y tenían bajos ingresos económicos. Un hallazgo positivo del estudio de Choy fue que los estudiantes en riesgo de abandonar la universidad o de obtener bajas calificaciones, una vez se graduaban tenían iguales oportunidades de empleo y salario que aquellos cuyos padres poseian estudios universitarios.

Según estudios, la primera generación de estudiantes en acudir a la universidad tiende a obtener bajas calificaciones y presentar mayores dificultades en el ajuste (Brown, 2008; Núñez \& Cuccaro, 1998). Estos estudiantes perseveran menos en los estudios, tienen bajos niveles de integración social e ingresos, y estudian a medio tiempo; además, seleccionan la universidad que les ofrezca ayuda financiera y la posibilidad de terminar pronto los estudios.

La Universidad de Puerto Rico es la principal institución pública del pueblo puertorriqueño que promueve el reclutamiento de estudiantes talentosos, mayormente provenientes de familias de escasos recursos socioeconómicos; por esta razón, no es de extrañar que ingresen estudiantes que correspondan a la primera generación de hijos que realizan estudios universitarios. Además, al ser esta institución universitaria la más prestigiosa del país, también existe la tendencia a que sea mayor la demanda de estudiantes 
provenientes de las diferentes clases sociales y de estudiantes cuyos padres han realizado estudios universitarios. Según hemos discutido, la literatura señala algunas variables sociodemográficas de los padres que pueden estar relacionadas al logro académico de sus hijos, como lo son su educación, estado civil, dificultades familiares y la disposición para involucrarse en actividades académicas y apoyar a sus hijos, por lo que resulta importante explorar estos temas para conocer las posibles necesidades de esta población estudiantil y atenderlas de forma más efectiva. Debido a que estudios señalan que la primera generación de estudiantes es la más propensa a fracasar, debe ser prioritario que los funcionarios de la universidad estén alertas a detectar proactivamente las diversas dificultades que pueda confrontar esta población. Tampoco se debe ignorar que vivimos en una sociedad cambiante y no necesariamente las dificultades y retos que confrontaron en su juventud y en la universidad los padres que realizaron estudios universitarios son las mismas que experimentan sus hijos hoy día. Por lo tanto, aunque los padres tengan la buena voluntad de apoyar, ayudar $y$ comprender a sus hijos universitarios, al desconocer el ambiente al que éstos se enfrentan, se les hace difícil entender las tensiones que éstos experimentan.

Investigadores expertos en la conducta y necesidades de los universitarios y nuestra experiencia en la ejecutoria profesional sugieren que además de ofrecer una semana de orientación a los estudiantes de nuevo ingreso, ésta debe extenderse también a sus padres de manera que se les faciliten técnicas y estrategias para ayudar a sus hijos. Ésta es una manera de reconocer a los padres como la principal red de apoyo de los estudiantes a la vez que se les conciencia de su responsabilidad de integrarse y participar en la promoción del rendimiento académico y desarrollo personal de sus hijos.

\section{Objetivos Generales}

1. Desarrollar un perfil socio demográfico de los padres de los estudiantes de Nuevo Ingreso del Año Académico 2009-2010.

2. Desarrollar un perfil socio demográfico de los estudiantes de Nuevo Ingreso del Año Académico 2009-2010.

3. Conocer las necesidades de los padres de los estudiantes de Nuevo Ingreso del Año Académico 2009-2010.

4. Conocer la condición de salud de los estudiantes de Nuevo Ingreso del Año Académico 2009-2010.

5. Conocer el interés de los padres de beneficiarse de talleres de capacitación para ayudar a sus hijos a manejar 
dificultades, promoviendo así la retención estudiantil y evitando el fracaso académico de los estudiantes de Nuevo Ingreso.

6. Conocer temas de interés de los padres y sus prioridades los cuales sirvan de guía al momento de planificar talleres de orientación o desarrollo personal dirigidos a los padres de los estudiantes de nuevo ingreso.

\section{Método}

\section{Selección de la Muestra y Procedimiento}

Se seleccionaron todos los padres de los estudiantes de Nuevo Ingreso que acudieron con sus hijos a las actividades de orientación programadas por el Decanato de Estudiantes para los estudiantes de Nuevo Ingreso y sus padres los días 13, 14 y 15 de julio de 2009. A todos los padres que asistieron a dicha actividad se les brindó la misma oportunidad de participar de esta investigación en carácter voluntario. El instrumento se distribuyó individualmente a cada uno de los padres que aceptaron participar, y se les orientó sobre los objetivos del estudio.

El cuestionario diseñado para tales efectos fue contestado individualmente por los participantes. Se logró distribuir 347 cuestionarios de los cuales 336 fueron contestados y entregados. Se esperaba la participación de un mínimo de 250 participantes (50 varones y 200 féminas) lo que sobrepasa las expectativas para obtener una muestra representativa.

\section{Instrumento de Medición}

Se elaboró un cuestionario el cual fue validado siguiendo los indicadores para instrumentos de investigación de esta categoría de estudios. Su diseño fue variado y constó de dos partes con un conjunto de preguntas cerradas y abiertas. La primera parte del instrumento contenía cuatro reactivos relacionados con datos personales y socio demográficos de los padres, a saber: relación con el estudiante de nuevo ingreso, estado civil, preparación académica y escuela de procedencia. En la segunda parte del instrumento, los reactivos del 1 al 11 contenían datos personales y socio demográficos de sus hijos. Los reactivos 12 y 13 se relacionaban a la disponibilidad de los padres para asistir a talleres y/o conferencias sobre temas relacionados con sus hijos universitarios. El reactivo 14 presentaba la oportunidad de seleccionar de entre nueve temas aquellos que les pudiera interesar que se cubrieran en talleres posteriores. Presentaba además una opción designada como "otros" para añadir otros temas que no estuvieron mencionados en la lista presentada y que fueran de su interés. 


\section{Tipo de Estudio y Análisis Estadístico}

Este estudio es exploratorio, aunque tiene elementos de estudios explicativos, descriptivos y correlacionales. Se considera exploratorio porque en la revisión de literatura no se encontraron estudios hechos a la misma población, sino ideas vinculadas pero aún no específicas sobre el tema investigado. En este estudio se obtuvo información específica para generar datos, lo cual permitió obtener un perfil socio demográfico de los sujetos, según se desprende de la primera parte del instrumento de investigación y parte de la segunda. Tiene aspectos descriptivos, ya que se logró especificar las propiedades que se consideraron más importantes de la población estudiada. Se seleccionaron una serie de reactivos y se midieron cada uno de estos independientemente.

La segunda parte del instrumento contiene reactivos dirigidos a explorar distintas variables en sujetos investigados. Además, este estudio midió y describió el grado de relación entre algunas variables, lo cual es característica de estudios correlacionales, descriptivos y que tienen valor específico. Se integraron en este estudio las mediciones de cada una de las variables para describir cómo se manifiestan y cómo se relacionan con la mayor precisión posible.

El análisis estadístico se llevó a cabo con el programa computadorizado denominado Statistical Package for the Social Sciences (SPSS - Programado Estadístico para las Ciencias Sociales).

\section{Hallazgos y Discusión}

Los hallazgos obtenidos en esta investigación cumplieron con los objetivos propuestos. Se logró obtener un perfil socio demográfico de los estudiantes de nuevo ingreso bajo estudio y sus padres, conocer sobre las condiciones de salud de los hijos, las dificultades que experimentan con ellos, su interés en beneficiarse de talleres de capacitación y sus prioridades en torno a temas de interés.

El porcentaje mayor $(72 \%)$ de los padres que participaron en esta investigación y que acudieron a las actividades de los estudiantes de nuevo ingreso correspondió a las madres $(\mathrm{N}=242)$, seguido por un $21.4 \%$ de asistencia de los padres $(\mathrm{N}=72)$; además hubo un $3.0 \%$ que correspondió a otros familiares como abuelas, tíos y primos $(\mathrm{N}=10)$. Esto guarda relación con los patrones que imperan en la cultura latina, especialmente la nuestra, en el que las madres han demostrado un rol más activo de participación en las actividades educativas de sus hijos mientras que, por lo general, los padres tienden a delegar en ellas esta responsabilidad. Siguiendo los planteamientos de Tinto (1999) y Carter (2006), estos estudiantes podrían estar más inclinados a 
alcanzar un mejor ajuste en la universidad ya que la participación de los padres en las actividades de sus hijos guarda relación con un ajuste emocional estable y un rendimiento académico exitoso.

El $62.5 \%$ de los padres eran casados $(\mathrm{N}=210), 21.4 \%$ divorciados $(\mathrm{N}=72), \quad 9.2 \%$ solteros $(\mathrm{N}=31)$ y un $1.8 \%$ viudos $(\mathrm{N}=6)$. El resto de los participantes indicaron que convivían con su pareja, estaban separados y otros no especificaron su status civil. Se puede deducir que el porcentaje mayor $(62.5 \%)$ de los hijos de la población estudiada convive con ambos padres $(\mathrm{N}=210)$. Si estos jóvenes mantienen buenas relaciones con su familia, son los que según Ruther (1999), están propensos a mantenerse en la universidad porque pueden recibir de sus padres apoyo, ayuda en sus dificultades personales y en las decisiones acerca de sus carreras. Sin embargo, aquellos cuyos padres están divorciados, viudos o separados, es muy probable que hayan estado expuestos a las dificultades que confrontaron sus padres durante sus procesos de separación, divorcio o viudez. Estos jóvenes son los que muchas veces vienen a la universidad con reacciones tensas hacia sus familias por estos aspectos, entre otros (Inghram, 1998) y presentan menor ajuste en la universidad (Carter, 2006). Además, es necesario considerar que las tasas de divorcio en Puerto Rico han ido aumentando (Departamento de Salud, 2007), por lo que es probable que cada vez sean más los estudiantes afectados que requieran servicios de un profesional de ayuda para lidiar con este proceso.

El porcentaje mayor $(72.3 \%)$ de los padres estudió en escuelas públicas $(\mathrm{N}=243)$ y un $23.8 \%$ en escuelas privadas $(\mathrm{N}=80)$. Solamente un $1.8 \%$ indicó que había estudiado en ambas $(\mathrm{N}=6)$. De esta población, el $13.1 \%$ alcanzó escuela superior $(\mathrm{N}=44)$ y el resto $(\mathrm{N}=292)$ había realizado estudios universitarios entre bachilleratos $(45.2 \%)$, grados asociados $(19.9 \%)$, maestrías $(16.1 \%)$, doctorados $(3.6 \%)$ y postgrados $(2.1 \%)$. Se destaca el hecho de que el porcentaje mayor $(86.9 \%)$ de los padres posee estudios universitarios, dato que concurre con las estadísticas de los padres de estudiantes en los Estados Unidos (Students, 2006) donde se observa una tendencia de los padres a poseer más estudios graduados que generaciones de padres anteriores (Daniel, Evans, \& Scott, 2001). Por tanto, la mayoría de los estudiantes no eran de primera generación y podrían tener un mayor logro académico si se considera que puede haber una relación entre la educación de los padres y el logro académico de sus hijos (Horn \& Kojaku, 2001; Steinmayr et al., 2010).

Los estudiantes de primera generación son más propensos a obtener bajas calificaciones, perseverar menos en los estudios, y tener bajos niveles de integración social y ajuste (Brown, 2008; 
Choy, 2002). . Mientras que aquellos estudiantes cuyos padres fueron a la universidad pueden manejar más efectivamente los retos de la vida universitaria resultando en un mejor ajuste, una mayor retención y tasas más altas de graduación. Sin embargo, al enfrentarnos a una sociedad tan cambiante, no necesariamente las dificultades que experimentan los jóvenes universitarios del siglo XXI son las mismas que experimentaron sus padres y las alternativas para afrontarlas también pueden variar dependiendo de los recursos de que dispongan. He ahí por lo tanto la necesidad de capacitar a los padres para que puedan ayudar a sus hijos a afrontar sus dificultades a tono con los tiempos que les han tocado vivir.

De los hallazgos se desprende que existe diferencia entre la escuela de procedencia de los padres y la de los hijos. El porcentaje mayor de los padres $(72.3 \%)$ estudió en escuelas públicas $(\mathrm{N}=243)$, mientras que el porcentaje mayor de los hijos $(58.9 \%)$ estudió en escuelas privadas ( $\mathrm{N}=198)$. El $39.6 \%$ de los hijos estudió en escuelas públicas $(\mathrm{N}=133)$, un $0.3 \%$ en escolarización en el hogar $(\mathrm{N}=1)$ y un $0.3 \%$ no especificó $(\mathrm{N}=1)$. Al poseer la mayoría de los padres estudios universitarios, su condición económica y las oportunidades de empleo pudieron haber mejorado, lo que a su vez los coloca en mejor posición de costear escuelas privadas para sus hijos. En Puerto Rico, gran parte de la población considera que los colegios privados ofrecen mejor calidad de enseñanza y preparan mejor a los estudiantes para ingresar a la universidad; es así que los padres que tienen los recursos económicos tienden a enviar a sus hijos a estas instituciones. Generalmente los estudiantes de familias de escasos recursos económicos son los que acuden a las escuelas públicas del país. De otra parte, en los últimos años ha surgido la tendencia de educar a los hijos en el hogar, lo cual se refleja en este estudio ya que el $0.3 \%$ de los estudiantes recibieron escolarización en el hogar.

En base a lo reportado por los padres, el porcentaje mayor de los estudiantes $(41.1 \%)$ aspira a realizar estudios doctorales $(\mathrm{N}=131)$, a diferencia de sus padres donde solamente un $3.6 \%$ posee estudios doctorales $(\mathrm{N}=12)$. Un $34.5 \%$ aspira a un bachillerato $(\mathrm{N}=116)$ y un $22.9 \%$ aspira a una maestría $(\mathrm{N}=77)$. Por consiguiente, es mayor la cantidad de estudiantes que aspira a estudios posgraduados ( $\mathrm{N}=215)(64 \%)$. Esto coincide con las estadísticas de universidades estadounidenses donde la mayoría de los estudiantes planifican realizar estudios posgraduados. No obstante, se observa una diferencia en términos de que la mayoría de los estudiantes estadounidenses aspiran a obtener una maestría en lugar de un doctorado (Students, 2006). Mas es necesario señalar que, al ser los padres quienes ofrecieron la información en nuestro estudio, no 
necesariamente corresponde a los planes reales de sus hijos, sino que podrían reflejar las expectativas de los padres.

En ocasiones, las discrepancias entre padres e hijos sobre expectativas y logros académicos pueden afectar adversamente las relaciones con los progenitores. En consecuencia, uno de los aspectos que más le preocupa a los estudiantes es la presión que reciben o perciben de sus padres al no poder obtener las calificaciones esperadas o alcanzar sus metas de estudios (Agliata, 2005; Agliata \& Renk, 2008; Whitaker \& Slimak, 1993; Whitaker, 1996). El porcentaje mayor $(61.9 \%)$ de los hijos de los padres encuestados eran mujeres $(\mathrm{N}=208)$, lo cual guarda relación con la población estudiantil de este Recinto en el que la mayoría de las estudiantes son féminas. Según los datos de la Oficina de Planificación Académica (OPA), para el año 20092010 ingresaron 1804 féminas (62.9\%) en comparación con 1064 varones $(37.1 \%)$, por lo que el hallazgo de este estudio es casi igual al de OPA. Esta mayor proporción de féminas realizando estudios ha sido observada también en Estados Unidos así como en países latinos (Bonilla, López, Cintrón, Ramírez, \& Román, 2005; Mather \& Adams, 2007; Students, 2006). Las edades, de ambos sexos, se encuentran en su mayoria entre los 18 (28.9\%) y $17(26.8 \%)$ años ( $\mathrm{N}=187)$, mientras que las cifras de OPA indican que el $96.4 \%$ de los estudiantes de nuevo ingreso tenían 17 años o menos. Esta discrepancia se puede deber en parte a que un $42.3 \%$ de los padres no contestó esta pregunta. Al observar la edad de la mayoría de los estudiantes se puede deducir que han ingresado inmediatamente después de haber concluido su educación secundaria y que están o han sido dirigidos a reconocer una gran importancia a la educación universitaria en la formación de un adulto competente y exitoso en la vida. Implica a su vez que se encuentran en una etapa de transición de adolescente a adulto joven lo cual conlleva otros retos para el estudiante, además de enfrentarse a su proceso de ajuste a la vida universitaria.

Se encontró que el porcentaje mayor (33.3\%) de los hijos de la población bajo estudio entró por la Facultad de Ciencias Naturales $(\mathrm{N}=112)$, hallazgo que coincide con los datos de OPA al ser la Facultad de Ciencias Naturales la que más estudiantes tuvo de nuevo ingreso para ese año académico. De éstos el $68.2 \%$ estudió en escuelas privadas $(\mathrm{N}=73)$ y el $33 \%$ en escuelas públicas $(\mathrm{N}=37)$. El porcentaje mayor $(72.1$. \%) de esta facultad correspondió al sexo femenino $(\mathrm{N}=80)$. Los otros porcentajes de ingreso correspondieron a la facultad de Administración de Empresas $(\mathrm{N}=61)$ con un $18.2 \%$, Ciencias Sociales $(\mathrm{N}=41)$ con $12.2 \%$, Educación $(\mathrm{N}=39)$ con $11.6 \%$, Humanidades $(\mathrm{N}=34)$ con $10.1 \%$, Comunicación Pública $(\mathrm{N}=19)$ con $5.7 \%$, Arquitectura 
$(\mathrm{N}=13)$ con $3.9 \%$, Estudios Generales $(\mathrm{N}=5)$ con $1.5 \%$ y no hubo contestación de un $3.6 \%$ de los participantes $(\mathrm{N}=12)$. Cabe destacar que a diferencia de Administración de Empresas, donde el porcentaje mayor de estudiantes $(53.2 \%)$ correspondió al sexo masculino ( $\mathrm{N}=33)$, en el resto de las otras facultades mencionadas el porcentaje mayor correspondió a las féminas. Al comparar con los datos de OPA, se observa un predominio de las féminas en todas las facultades documentadas en este estudio, incluyendo la de Administración de Empresas.

Solamente el $17.9 \%$ de los padres contestaron que sus hijos tienen alguna condición de salud $(\mathrm{N}=60)$. De la muestra total, el $13.1 \%$ reportó condiciones de salud física $(\mathrm{N}=44)$, y el $3.6 \%$ emocional ( $\mathrm{N}=12)$. Hubo un $1.2 \%$ que contestó que sus hijos tienen ambas condiciones de salud, es decir físicas y emocionales $(\mathrm{N}=4)$ e indicaron que el $14.0 \%$ recibe tratamiento para su condición de salud $(\mathrm{N}=47)$ y el $3.9 \%$ no $(\mathrm{N}=13)$. Esta población de estudiantes son los posibles candidatos a beneficiarse de los programas de ayuda que ofrece el Decanato de Estudiantes de manera que no se afecte su rendimiento académico por su condición de salud; entre éstos se encuentran el Departamento de Consejería para el Desarrollo Estudiantil, la Oficina de Ayuda a Personas con Impedimentos y el Departamento de Servicios Médicos.

Los hallazgos indican que un $11.3 \%$ de los padres está confrontando dificultades con sus hijos ( $\mathrm{N}=38)$ y un $7.4 \%$ indicó que está recibiendo ayuda ( $\mathrm{N}=15)$. Además, el reactivo 10 del cuestionario era una pregunta abierta en la que se solicitó a los participantes que señalaran las dificultades que estaban confrontando con sus hijos y éstas fueron sus respuestas en orden de prioridad: dificultades familiares, económicas, comunicación, hostilidad, conducta agresiva, rebeldía y pérdida del tiempo. Cabe la posibilidad de que los estudiantes que están experimentando dificultades con sus padres y que no están recibiendo ayuda para manejarlas sean los más propensos a fracasar académicamente ya que, además de enfrentarse a los retos que conlleva el ajuste a la vida universitaria, traen otras fuentes de tensión que les restan energía y concentración necesarias para lidiar con esta nueva etapa en sus estudios. Por ello resulta imperativo el procurar mayor contacto con estos estudiantes por parte del personal de la universidad para que se trabajen estas y otras dificultades que puedan afectar su desempeño. Es responsabilidad de los profesionales que laboramos directamente con ellos el desarrollar actividades y programas donde podamos ayudar tanto a los estudiantes como a su familia inmediata en el fortalecimiento y/o desarrollo de destrezas de afrontamiento ante estas dificultades. Asimismo, 
es necesario que el personal esté capacitado debidamente para atender las mismas y que se mantenga al tanto de las tendencias y necesidades al asistir con regularidad a talleres y conocer los hallazgos de las investigaciones que se realizan.

Al encuestar a los padres sobre su disponibilidad para asistir a talleres de capacitación, el porcentaje mayor (95.8\%) manifestó interés $(\mathrm{N}=322)$. De éstos, un $58.9 \%$ prefiere que los talleres sean los sábados ( $\mathrm{N}=198)$ y un $29.5 \%$ los viernes $(\mathrm{N}=99)$. El resto de la población se distribuyó entre los otros días de la semana. Estos resultados podrían reflejar el compromiso de los padres en capacitarse para el manejo de dificultades con sus hijos, su necesidad de fortalecer destrezas para manejar situaciones con ellos y su disponibilidad en brindarles apoyo. Según Hummel y Steele (1996), los estudiantes que reciben apoyo y ayuda de sus padres en el logro de sus metas académicas, no sólo perseveran sino que experimentan un intenso deseo de sobresalir en los estudios y de superar su nivel de vida.

De otra parte, los seis temas principales que a los padres les interesa que se cubran en los talleres de capacitación, de acuerdo a las alternativas presentadas en el cuestionario, corresponden a: Orientación sobre ayudas a los estudiantes $(61.9 \%)(\mathrm{N}=208)$, Métodos y maneras de ayudarlos en sus responsabilidades universitarias $(58.6 \%)(\mathrm{N}=197)$, Retos, barreras y dificultades $(58.0 \%)(\mathrm{N}=195)$, Ajuste a la vida universitaria $(52.1 \%)(\mathrm{N}=175)$, Comunicación asertiva $(50.5 \%)(\mathrm{N}=170)$ e Independencia personal $(50.0 \%)(\mathrm{N}=168)$. Se observa que el tema que más les interesa es el de Orientación sobre ayudas, lo cual podría contribuir a lidiar con las principales dificultades señaladas por los padres como lo son las familiares y las económicas. Así, es fundamental el informar tanto a padres como a estudiantes sobre las diversas ayudas que existen para que puedan beneficiarse de éstas. De no ser así, es menos probable que los estudiantes accedan a servicios como los de consejería y psicoterapia (Yorgason, Linville, \& Zitzman, 2008).

Cabe destacar que en el reactivo 14 del cuestionario se les solicitó indicar otros temas de interés y éstas fueron las respuestas de algunos de los sujetos en orden de prioridad: comunicación, seguridad dentro y fuera de la universidad, responsabilidades paternales, cómo ayudar a los hijos en destrezas de estudios, manejo de crisis, cómo vencer la timidez y autoestima. Estos resultados nuevamente señalan la necesidad de los padres en prepararse para atender situaciones que puedan enfrentar sus hijos durante el transcurso de su vida universitaria y la necesidad de trabajar con las destrezas de comunicación en la familia; además, responden a la realidad de nuestro contexto al dar mucha importancia a asuntos de seguridad debido a la criminalidad en el pais y en la misma universidad. 
El tema de la comunicación fue uno de los más señalados en la pregunta abierta sobre temas de interés. Argumenta Tinto (1999) que en gran medida la separación de la familia y la transición e integración de los estudiantes a la universidad son exitosas dependiendo del apoyo que reciban de sus padres. Esto es sustentado por Agliata (2005) quien recomienda el realizar actividades educativas para los padres de los estudiantes de nuevo ingreso y propiciar su participación de manera que se involucren en apoyar a sus hijos en el proceso de ajuste a la institución. En años recientes, el DCODE ha incorporado talleres educativos para los padres en las actividades de orientación de los estudiantes de nuevo ingreso con el objetivo de integrarlos en la vida universitaria de sus hijos a la vez que se les capacita para que puedan manejar las dificultades que puedan afrontar con ellos a lo largo de su vida estudiantil. En una de esas actividades fue donde se logró recopilar los datos de este estudio.

\section{Limitaciones y Recomendaciones}

El instrumento para la recopilación de datos fue validado; sin embargo, no se encontraron instrumentos para este tipo de estudios validados por otros investigadores que sirvieran de base para el diseño del mismo. Los hallazgos de esta investigación solamente corresponden a los participantes bajo estudio, por lo que no se establecen generalizaciones a todos los padres de los estudiantes de nuevo ingreso. A pesar de que se logró una muestra representativa, hubo días en los que la cantidad de padres que asistió a las actividades de orientación fue limitada debido a que la carta de invitación de las facultades, a excepción de Ciencias Naturales, no les llegó a tiempo.

Se recomienda dar a conocer los resultados de este estudio a los funcionarios que brindan servicios a la población estudiantil de manera que conozcan las necesidades y el perfil socio demográfico de los padres de los estudiantes de nuevo ingreso. Además, dar a conocer estos mismos resultados a los Docentes de la Consejería de los once Recintos del Sistema de la Universidad de Puerto Rico de manera que les siva de base para la selección de los temas al momento de planificar talleres de capacitación para los estudiantes de nuevo ingreso. Finalmente, realizar otras investigaciones en torno al mismo tema de manera que se puedan ir generalizando y depurando los resultados de estos estudios y se logre tener un conocimiento más preciso de las necesidades y perfil socio demográfico de los padres de los estudiantes de nuevo ingreso. Sería interesante el realizar estudios donde se explore el aprovechamiento académico en relación a las diferentes variables que pudieran haber influenciado positiva o negativamente el mismo y en relación a las ayudas recibidas por los estudiantes. 


\section{Referencias}

Agliata, Allinson Kanter. (2005). College students' well being: The role of parental college student expectation discrepancies and communication. (Disertacón doctoral). Disponible en ProQuest Dissertation and Theses. (UMI No.3196617).

Agliata, Allison Kanter, \& Renk, Kimberly. (2008). College stdents' adjustment: The role of parent-college student expectation discrepancies and communication reciprocity. Journal of Youth Adolescence, 17, 967-982. doi: 10.1007/s10964-007-9200-8

Astin, Alexander, W. (1997). The conditions of America liberal learning: Pragmatism and challenging traditions. New York: The College Board Press.

Bonilla, Víctor E., López, Annette, Cintrón, Madelyn E., Ramírez, Solángel, \& Román, Rosa. (2005, diciembre). Feminización de la matrícula de educación superior en Puerto Rico. Cuaderno de Investigación en la Educación, 20. Recuperado de http://cie. uprrp.edu/cuaderno/ediciones/20/pdf/c20art7.pdf

Brown, Natashia. (2008). Predicting college adjustment: The contribution of generation status and parental attachment. (Disertación doctoral). Disponible en ProQuest Dissertation and Theses (UMI No. 3332978).

Campbell, Lisa B. (2004). Factors predicting adjustment to college: A longitudinal study. (Disertación doctoral). Disponible en ProQuest Dissertations and Theses. (UMI 3164679).

Carter, Elizabeth. (2006). Parental assistance and first year college student independence and adjustment. (Disertación doctoral). Disponible en ProQuest Dissertations and Theses (UMI3241848).

Choy, Susan, P. (2002). Students whose parents did not go to college: Postsecondary access, persistence and attachments. National Center for Education Statistics, U.S. Department of Education. Washington, D.C., EE. UU. : U.S. Government Printing Office.

Daniel, Bonnie, V., Evans, Scott G., \& Scott, B. Ross. (2001). Understanding family involvement in the college experience today. New Directions for Student Services, 4, 3-13.

Departamento de Salud. Estado Libre Asociado de Puerto Rico. (2007). Divorcios concedidos en las salas del Tribunal Superior por región y causal. Recuperado de http://www. salud.gov.pr/Datos/InfoSalud/Statistics/Divorcios/Pages/ DIVORCIOSCONCEDIDOSENLASSALASDELTRIBUNALSUP ERIORPORCAUSALYSALAPUERTORICO2005.ASPX

De Jesús, Juan (1988). Rendimiento académico de los estudiantes suspendidos por deficiencia académica del Recinto de Río Piedras al inicio del primer semestre del año académico 1987-1988: Departamento de Consejería para el Desarrollo Estudiantil, Decanato de Estudiantes, Recinto de Río Piedras, Universidad de Puerto Rico. Proyecto de investigación no publicado. 
García, Mercedes. \& San Segundo, Jesús. (2002). El rendimiento académico en el primer curso universitario. Jornadas de la Asociación de Economía de la Educación. España, 435-445.

Guemarez, Carmen. (1998). Jóvenes víctimas de violencia en la relación de pareja. (Tesis de maestría no publicada).Universidad de Puerto Rico, Recinto de Río Piedras.

Hermann, Lauren. (2007). Adjustment to college: The role of optimism, coping strategies, and parental attachment. (Disertación doctoral). Disponible en ProQuest Dissertations and Theses . (UMI 3287857).

Horn, Laura, \& Kojaku, Lawrence K. (2001). High school academic curriculum and resistance path through college. National Center for Educational Statistics. U. S. Department of Education. Washington, DC: U.S. Government Printing Office. Recuperado de http://nces.ed.gov/pubs2001/2001163.pdf

Hummel, Mary. \& Steele, Claude. (1996). A program to address issues of academic achievement and retention. Journal of Intergroup Relations, 33(2), 28-33.

Inghram, Christine. (1998). Los ataques de pánico. Barcelona, España: Oniro

Kenny, Maureen, \& Rice, Kenneth. (1995). Attachment to parents and adjustment in late adolescent college students. The Counseling Psychologist, 23(3), 433-456.

Mather, Mark., \& Adams, Dia. (2007). The crossover in femalemale college enrollment rates. Population Reference Bureau. Recuperado de: http://www.prb.org/Articles/2007/ CrossoverinFemaleMaleCollegeEnrollmentRates.aspx

Mohammdi, John. (1996, primavera). Exploring retention and attrition in a two years public community college. VCCA Journal, 10(1), 3950.

Navas, José. (1998). Conceptos y Teorías de Aprendizaje: Publicaciones Puertorriqueñas.

Núñez, Ann-Marie, \& Cuccaro-Alamin, Stephanie. (1998). First generation students: Undergraduates whose parents never enrolled in postsecondary education. National Center of Educational Statistics. U.S. Department of Education, Washington, DC, EE. UU: Government Printing Office.

Oficina de Planificación Académica. (2010). Libro de datos estadísticos 2009-2010. Universidad de Puerto Rico, Recinto de Río Piedras. Recuperado de: http://opa.uprrp.edu/pls/opaweb/ factbook? anoacademico $=2009-2010$

Ostolaza, Margarita. (2001). La Transformación de la Educación Superior en Puerto Rico: Comisión de Educación, Ciencia y Cultura del Senado de Puerto Rico.

Palladino Schulthiss, Donna E., Kress, Helen M., Manzi, Alberta J., \& Jeffrey Glasscock, Julie M. (2001). Relational influence in career development: A qualitative inquiry. The Counseling Psychologist, 29(2), 214-239. 
Puente, Anibal. (1999). El cerebro creador. Madrid, España: Alianza Editorial, S.A.

Rivera, Nitza. (2003). Reserva Histórica. Manual Informativo de Servicios al Estudiante. Decanato de Estudiantes, Recinto de Río Piedras, Universidad de Puerto Rico: University Press.

Ruther, Michael. (1999). Resilience concepts and findings: Implications for family therapy. Journal of Family Therapy, 21, 119-144.

Steinmayr, Ricarda, Dinger, Felix C., \& Spinath, Birgit. (2010). Parents' education and children's achievement: The role of personality. European Journal of Personality, 24, 535-550. doi: 10.1002/ per.755

STUDENTS. (2006). Chronicle of Higher Education, 53(1), 12-22. Disponible en Academic Search Complete.

Universidad de Puerto Rico (1998-1990). Plan Estratégico de Acción del Recinto de Río Piedras de la Universidad de Puerto Rico, Certificación 67, Río Piedras, Puerto Rico: University Press.

Whitaker, Leighton. \& Slimak, Richard, E. (1993). College Student Development. Nueva York: Haworth Press.

Whitaker, Leighton. (1996). Treating students with personality disorders: A costly dilemma. Journal of College Student Psychotherapy, 10(3), 29-44.

Yorgason, Jeremy B., Linville, Deanna, \& Zitzman, Bryan. (2008). Mental health among college students: Do those who need services know about and use them? Journal of American College Health, 57(2), 173-181. 